\title{
MORSE THEORY APPLIED TO $N=1$ AND 2 SUPERCONFORMAL THEORIES
}

\author{
Caren MARZBAN \\ International Centre for Theoretical Physics, I-34100 Trieste, Italy
}

Received 11 December 1989

\begin{abstract}
Various spaces are singled-out as candidates for the space of all 2D $N=1$ and 2 supersymmetric quantum field theories, respectively. This is done by treating the $c$-function as a Morse function on these spaces.
\end{abstract}

\section{Introduction}

The study of string theory and that of critical phenomena in two dimensional systems has made the plan of the classification of conformal field theories (CFTs) one of paramount importance. However, the concept of classification is not one that renders itself to a precise definition. The latest program, which in a self-evident way qualifies to be called one of classification, is based on singularity theory [1]. Another, motivated mostly by the hope of gaining some understanding of non-perturbative phenomena in string theory, is the idea of treating CFTs as "special points" in the space, $\mathrm{Q}$, of all 2D quantum field theories (QFTs) [2]. Although different in approach, there exist certain common grounds in these two ideas. For instance, renormalization group ( $R G$ )-flows play a central role in both strategies.

The space $\mathrm{Q}$ and the RG-flows are addressed in an elegant and unifying fashion in a theorem, due to Zamolodchikov [3], called the "c-theorem". In this theorem, in addition to advocating the existence of a function $c$, defined over $\mathrm{Q}$, whose critical points correspond to CFTs (i.e. fixed points of the RG-flow), one also demands that the RG-flows decrease the value of the function $c$. The setting in the $c$-theorem is one which is most suitable for the application of Morse theory. There, knowledge of the behaviour of a function, defined over a manifold, near its critical points provides one with a great deal of global (topological) information regarding the manifold itself. In this way we can learn about the space $Q$, solely from the knowledge of the $c$-function defined on it.

The subspace, $\mathrm{Q}_{0} \subset \mathrm{Q}$, corresponding to $N=0$ unitary CFTs (i.e. $c<1$ ) was treated in this way by Vafa [4] and, with a slightly different emphasis, by Das et al. [5]. Mostly in the spirit of the former paper, in this letter we apply Morse theory to $N=1$ and $N=2$ unitary super-CFTs (i.e. $c<\frac{3}{2}$ and $c<3$, respectively). This will allow us to explore the topology of a larger subspace of $Q$ than that of $Q_{0}$. We will be cavalier in our handling of the space $\mathrm{Q}$; in addition to the various technicalities which arose in the $N=0$ case (which were addressed in ref. [4]), the $N=1$ and 2 cases call for further "restrictions" on the relevant subspaces of $Q$, which will be discussed below.

\section{Morse theory and $N=0$ CFTs}

In this section we briefly recall elementary ideas from Morse theory, and review its application to the $N=0$ case, as treated by Vafa [4].

First, let us develop what is often referred to as "baby Morse theory" [6]. Given a compact manifold $\mathrm{M}$, and a non-degenerate function $f$ defined on it, one defines a Morse polynomial (MP) for $f$ by $M(f ; t) \equiv \sum_{P_{i}} t^{n_{i}}$, where $P_{i}$ are the critical points of $f$, at which $n_{i}$ is the index of $f$, i.e. the number of negative eigenvalues of the hessian (or, in other words, the number of directions in which $f$ decreases ). $t$ is an arbitrary parameter between 0 and 1. For the manifold $\mathrm{M}$, one defines a Poincaré polynomial $(\mathrm{PP}), P(\mathrm{M} ; t) \equiv \sum_{k=0}^{\operatorname{dim} \mathrm{M}} b_{k}(\mathrm{M}) t^{k}$, where $b_{k}(\mathrm{M}) \equiv$ 
$\operatorname{dim} \mathrm{H}^{k}(\mathrm{M})$ are the Betti-numbers of $\mathrm{M}$. Now, given the function $f$ the "weak" Morse inequalities place upper-bounds on the $b_{k}$ of $\mathrm{M}: P(\mathrm{M} ; t) \leqslant M(f ; t)$. The "strong" form of the inequalities, however, serves our purpose better. It states

$M(f ; t)-P(\mathrm{M} ; t)=(1+t) Q(t)$,

where the coefficients of $Q(t)$ are non-negative. Notice the following consequence of $(1)$ :

$M(f ;-1)=P(\mathrm{M} ;-1)$.

The "lacunary principle" [6] states that if the product of all the consecutive coefficients in $M(f ; t)$ are zero, then $M(f ; t)=P(\mathrm{M} ; t)$. A function for which this equality holds is called a perfect Morse function. Thus, if one can find a function on $M$ whose MP satisfies the lacunary principle (i.e. a perfect function), then one can simply read-off the $b_{k}$ of $\mathrm{M}$ from the coefficients of $M(f ; t)$, or simply look up spaces which have $M(f ; t)$ as their PP.

Now, in ref. [4], $f$ and $M$ were identified with the $c$-function $c_{0}$ and the space $\mathrm{Q}_{0}$, respectively. Also, in that case, the critical values and the index of $c_{0}$ were nothing but $c(m)=1-6 /[m(m+1)]$ with $m \geqslant 2$, and the number of relevant operators (i.e. $2(m-2)$, excluding the identity) in the $N=0$ CFT, respectively. Then, $M\left(c_{0} ; t\right)=\sum_{m=2}^{\infty} t^{2(m-2)}=1+t^{2}+t^{4}$ $+\ldots=1 /\left(1-t^{2}\right)$. Since the lacunary principle does apply to this MP, $c_{0}$ is then perfect, i.e. the PP of the space $\mathrm{Q}_{0}$ is $P\left(\mathrm{Q}_{0} ; t\right)=1 /\left(1-t^{2}\right)$. Among the infinity of topologically distinct spaces which share this $\mathrm{PP}$, Vafa discussed (1) the loop-space of SU (2), and (2) $\mathrm{CP}^{\infty}$. Two more examples of such a space are (3) the space of all paths, joining two arbitrary points, on $\mathrm{S}^{3}$ and (4) the connected sum of $S^{2}, S^{4}, S^{6}, \ldots$ We shall generically refer to all these spaces, which are homologically equivalent, as $Q_{0}$, i.e. the space relevant to the $N=0$ unitary CFTs.

\section{Morse theory and the $N=1$ super-CFTs}

Let us now apply the paraphernalia of Morse theory to the $N=1$ superdiscrete series. The space under consideration is then the space of all $2 \mathrm{D} N=1$ supersymmetric QFTs, which we shall call $Q_{1}$. Of course, as in the $N=0$ case, we are assuming that the various technical difficulties regarding the application of
Morse theory (e.g. orbifold singularities, and the infinite-dimensional nature of $Q_{1}$, etc.) have been properly taken into account.

In computing the MP it is important to realize what the definition of "the index of the $c$-function" truly is. For the $N=0$ case, the index is simply the number of relevant fields in the CFT, since this is the number of RG-trajectories in the direction of which $c_{0}$ decreases. In the $N=1$ case, since we are considering RG-flows which preserve $N=1$ SUSY, the index of the $c$-function $c_{1}$ must be identified with the number of relevant super-fields (excluding the identity). Also note that since there are no SUSY-preserving marginal operators here, $c_{1}$ is non-degenerate. From refs. $[7,8]$ it can be seen that this number is $(m-2)$. Thus the MP for $c_{1}$ is

$M\left(c_{1} ; t\right)=\sum_{m=2}^{\infty} t^{m-2}=1+t+t^{2}+t^{3}+\ldots=\frac{1}{1-t}$.

In contrast to $c_{0}$, the perfectness of $c_{1}$ cannot be decided on the basis of the lacunary principle, since every power of $t$ is present. Thus, it is not possible to identify $Q_{1}$ (or the homology of $Q_{1}$ ) uniquely. However, Morse theory does provide us with restrictions on the possible Betti-numbers of $\mathrm{Q}_{1}$, as shown below.

The weak form of the inequalities already place limits on the $b_{k}-$ that $b_{0}\left(\mathrm{Q}_{1}\right)=1$, and $b_{k}\left(\mathrm{Q}_{1}\right)=0$ or 1 for $\forall k \neq 0$. The former relation implies that $\mathrm{Q}_{1}$ is connected. To further explore the possible allowed $0 / 1$ configurations of the $b_{k}\left(\mathrm{Q}_{1}\right)$, we must go on to consider the strong form of the inequalities.

The strong form of the Morse inequalities places strong restrictions on the possible values of the $b_{k}\left(\mathrm{Q}_{1}\right)$. Substituting (3) in (1) gives

$b_{k}=1-q_{k}-q_{k-1}$,

where for clarity of notation I have suppressed the $Q_{1}$ appearing in the argument of $b_{k}\left(\mathrm{Q}_{1}\right)$. Since (4) can be inverted,

$$
\begin{aligned}
q_{n} & =\sum_{k=0}^{n}(-1)^{k} b_{k} \quad \text { for } n=\text { odd } \\
& =\sum_{k=1}^{n}(-1)^{k+1} b_{k} \quad \text { for } n=\text { even }
\end{aligned}
$$

the non-negativeness of the $q_{k}$ allows us to develop an algorithm for enumerating all the possible $0 / 1$ 
configurations of the $b_{k}$. The result is as follows: If we indicate the 0 or 1 value of a $b_{k}$ by " $\left\{\begin{array}{l}0 \\ 1\end{array}\right.$ ", then in the sequence of $b_{k}$ (starting with $b_{0}=1$ ), any value is followed by a $\left\{\begin{array}{l}0 \\ 1\end{array}\right.$, except the 0 in $\left\{\begin{array}{l}0 \\ 1\end{array}\right.$ which is followed by a single 0 (not 1 ). This can best be seen, schematically, in the following scheme:

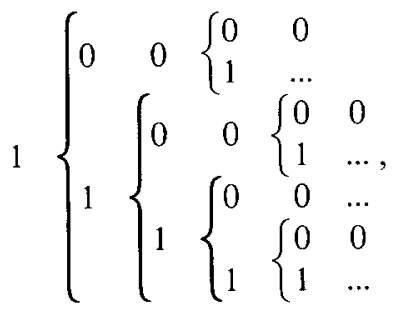

where from left to right, the columns correspond to the allowed values of $b_{0}\left(\mathrm{Q}_{1}\right), b_{1}\left(\mathrm{Q}_{1}\right), b_{2}\left(\mathrm{Q}_{1}\right)$, etc.

Although, at this stage we cannot enumerate all the possibilities, for there are infinite of them, we see that there are stringent restrictions on the allowed Bettinumbers. For example, as seen in the above scheme, spaces whose PP begin with $1+t^{2}$, or with $1+t+t^{3}$ are not allowed. The former implies that the $\mathrm{Q}_{0}$-candidates, in particular, are not legitimate as candidates for $Q_{1}$. The latter corresponds to examples of non-simply connected spaces which are again not allowed (however, see below).

Notice that as a result of (2) not all the solutions in the scheme are allowed. This equation places the following constraint on the $b_{k}$ :

$$
\sum_{k=0}^{\infty}(-1)^{k} b_{k}=\frac{1}{2} \text {. }
$$

However, it is important to realize that the evaluation of the infinite sum in $M\left(c_{1} ; t\right)$ (which, at $t=-1$ gives the $\frac{1}{2}$ in (6)) has already taken us into the realm of analysis. In other words, to impose (6) requires some sort of a "regularization" of the infinite sum on the left-hand side; recall that the $b_{k}$ are 0 or 1 . Intuitively, (6) means that the 0 s and the $1 \mathrm{~s}$ must be evenly distributed among the $b_{k}$ with $k=$ even and odd.

One way of "regularizing" the sum in (6) is by placing a cut-off on it. This amounts to treating $Q_{1}$ as a finite dimensional space - call it $\mathrm{Q}_{1}^{D}$. For our purposes it does make sense to treat $Q_{1}$ as finite dimensional, since we can consider only those super-CFTs (i.e. all the points of $\mathrm{Q}_{1}$ at which $c_{1}$ is equal to $c_{1}(m)=\frac{3}{2}[1-8 / m(m+2)]$ with $\left.m \geqslant 2\right)$ to which a given super-CFT $(m \neq \infty)$ flows; with $m=\infty$ removed, $Q_{1}$ can be finite dimensional.

In fact, for $Q_{1}^{D}$, we can discard the constraint (6) altogether, since it is nothing but a consequence of the Morse inequalities (at $t=-1$ ) which we have already taken into account. Instead, though, we have the Poincaré duality at our disposal (assuming $\mathrm{Q}_{1}^{D}$ is orientable ), i.e. $b_{k}=b_{D-k}$. Then, of the configurations appearing in the scheme, those which satisfy the Poincare duality have the following PPs: For $D=$ odd,

$$
\begin{aligned}
P\left(\mathrm{Q}_{1}^{D} ; t\right)= & 1+t^{D}, \\
= & 1+t+t^{D-1}+t^{D}, \\
= & 1+t+t^{2}+t^{D-2}+t^{D-1}+t^{D}, \\
& \vdots \\
= & 1+t+t^{2}+t^{3}+\ldots+t^{D},
\end{aligned}
$$

and for $D=$ even,

$P\left(\mathrm{Q}_{1}^{D} ; t\right)=1+t+t^{2}+t^{3}+\ldots+t^{D}$.

Before going any further, we can compute the Euler number of $\mathrm{Q}_{1}^{D}$ :

$$
\begin{aligned}
\chi\left(\mathrm{Q}_{1}^{D}\right) \equiv P\left(\mathrm{Q}_{1}^{D} ; t=-1\right) & =0 & \text { for } D=\text { odd } \\
& =1 & \text { for } D=\text { even } .
\end{aligned}
$$

For the $D=$ odd case, except for the first solution which is the PP of $S^{D}$, and for the last solution which we shall discuss below, the rest are difficult to identify with "common" spaces. Of course, to visualize their homology one can think in terms of connected sums of the $S^{N}$ (see below). Even without knowledge of the specific spaces, we can extract some useful information for $D=$ odd or even. For instance, all of our PPs have $b_{0}=1$, implying that $\mathrm{Q}_{1}^{D}$ is connected. Except for the case $\mathrm{Q}_{1}^{D}=\mathrm{S}^{D}$, we have $b_{1}=1$ which means that $\mathrm{Q}_{1}^{D}$ is in fact not simply connected - there exists exactly one non-contractible curve. In other words, $\mathrm{Q}_{1}^{D}$ has a hole in it. The Euler numbers (7) are also helpful in visualizing these spaces. A convenient representation which captures at least the homological structure of these spaces is given in terms of connected sums of $\mathbf{S}^{N}$. Corresponding to the various $D=$ odd solutions we have 


$$
\begin{aligned}
\mathrm{Q}_{1}^{D} \approx \mathrm{S}^{D}, \\
\approx \\
\approx \mathrm{S}^{1} \vee \mathrm{S}^{D-1} \vee \mathrm{S}^{D}, \\
\approx \mathrm{S}^{1} \vee \mathrm{S}^{2} \vee \mathrm{S}^{D-2} \vee \mathrm{S}^{D-1} \vee \mathrm{S}^{D}, \\
\quad \vdots \\
\approx \mathrm{S}^{1} \vee \mathrm{S}^{2} \vee \mathrm{S}^{3} \vee \ldots \vee \mathrm{S}^{D},
\end{aligned}
$$

and for the $D=$ even case we have only

$$
\mathrm{Q}_{1}^{D} \approx \mathrm{S}^{1} \vee \mathrm{S}^{2} \vee \mathrm{S}^{3} \vee \ldots \vee \mathrm{S}^{D},
$$

where " $\approx$ " indicates homological equivalence. The actual topology of $\mathrm{Q}_{1}^{D}$ may be quite different from these.

Finally, it is amusing that the only PP that appears in both the $D=$ even and odd case is $P\left(Q_{1}^{D}\right.$; $t)=1+t+t^{2}+t^{3}+\ldots+t^{D}$, which is equal to the MP itself, i.e. that $c_{1}$, as a function defined on this space, is perfect. A non-rigorous argument for the perfectness of $c_{1}$ would follow upon consideration of the $D \rightarrow \infty$ limit. In that limit the only consistent solution (i.e. common to both $D=$ even and odd) is

$$
\begin{aligned}
P\left(\mathrm{Q}_{1} ; t\right) & =\lim _{D \rightarrow \infty} P\left(\mathrm{Q}_{1}^{D} ; t\right)=1+t+t^{2}+\ldots \\
=\frac{1}{1-t} & =M\left(c_{1} ; t\right) .
\end{aligned}
$$

Thus, we see that, although the lacunary principle could not decide the perfectness of $c_{1}$, the MP for $c_{1}$ is special enough (i.e. with all coefficients equal to 1 ) so that one can at least entertain the possibility of the perfectness of $c_{1}$. Now, as an infinite dimensional space, other than $S^{1} \vee S^{2} \vee S^{3} \vee \ldots$, another candidate space for $Q_{1}$ is the space of paths, joining two arbitrary points, on $S^{2}$.

\section{Morse theory and the chiral $N=2$ super-CFTs}

$\mathrm{Q}_{2}$, the space of all 2D chiral, $N=2$ super-CFTs requires a slightly more careful analysis of the pattern of RG-flows. We have restricted our attention to the subspace of chiral theories, because it is these whose algebra's chiral rings have a correspondence with the local rings of the respective super-potentials, thereby equipping one with a rigorous geometric foundation [1]. Also, it is known that the unitary, chiral, theories have a Landau-Ginzburg (LG) description [1], which makes the study of RG-flows, and the matter of counting the number of relevant operators an easy task.

It turns out that the LG-superpotentials for chiral $N=2$ super-CFTs fall into the ADE pattern that appears in the classification of modality-zero, complex singularities [1]. This fact is more than sufficient to provide us with all the information we need for constructing the MP. Firstly, with the LG-superpotentials at hand, it is easy to see that, as in $N=0$ and 1 , RG-flows keep one within the A-series [9]. Therefore we are justified, again, to compute the MP for only the A-series. Secondly, the number of relevant chiral superfields (including the identity) is simply equal to the index of the singularity, as a result of the above-mentioned ring correspondence; for the case of the $\mathrm{A}_{k+1}$-series, with $k \geqslant 0$, this number is $(k+1)$ (or simply $k$, excluding the identity). Thirdly, the modality-zero condition, i.e. the absence of (SUSYpreserving) marginal operators, assures us that the $c$ function is non-degenerate. The final point is in regards to the complex nature of the superfield; as a result of this, the coupling constant in front of the relevant perturbation can also be complex. Recall that $\mathrm{Q}$, in general, can be considered a space of coupling constants, à la Zamolodchikov [7]. Thus, $Q_{2}$ is a complex space.

It is tempting to interpret the number of relevant chiral superfields, $k$, as the index of the $c$-function $c_{2}$. However, recalling that the index of a function is the number of negative eigenvalues of the hessian (i.e. a quadratic form ), the attempt of defining an "index" for a complex function, defined over a complex space, becomes hopelessly obstructed. This obstruction is essentially due to the fact that the field of complex numbers cannot be totally ordered $\#$. Although one can define critical points for a complex function, one cannot decide the maximum/minimum behaviour of the function at the critical points. In other words, whether $c_{2}$ increases or decreases, in a given (complex) direction, cannot be decided upon. This nonexistence of an analog of Morse theory, for complex spaces $\$ 2$, destroys all hope of learning about the

\#1 On this point, we acknowledge many fruitful discussions with M. Mahdavi-H.

\#2 There does exist an anolog of Morse theory for complex spaces, and it is called the Picard-Lefschetz theory (see ref. [10]). However, it lacks the analog of Morse inequalities. 
complex cohomology (in the sense of Dolbeault cohomology, $\mathrm{H}^{p, q}\left(\mathrm{Q}_{2}\right)$ ) of $\mathrm{Q}_{2}$.

The situation is however not entirely bleak, for since the value of $c_{2}$ at the critical points is real (this being $c_{2}(k)=3 k /(k+2)$ with $\left.k \geqslant 0\right)$, we can shift our attention from $\mathrm{Q}_{2}$ to its real "covering", $\mathrm{Q}_{2 \mathrm{R}}$. Indeed, since every compact complex ( $D$-dimensional) manifold is equivalent to a compact real ( $2 D$-dimensional) manifold, we can still apply Morse theory and extract information about the real (de Rham) cohomology of the space $Q_{2 R}$. In short then, we now have a real space, $Q_{2 R}$, and a real-valued $c$-function, $c_{2 \mathrm{R}}$, defined on it, available for the application of Morse theory. The only subtlety is that the index of $c_{2 R}$ at a critical point is not simply $k$ (see above), but rather $2 k^{\sharp 3}$, since at a given critical point there are now 2 independent real directions, associated to each relevant chiral superfield, in which $c_{2 \mathrm{R}}$ decreases.

Finally, the MP for $c_{2 \mathrm{R}}$ is $M\left(c_{2 \mathrm{R}} ; t\right)=\sum_{k=0}^{\infty} t^{2 k}=$ $1+t^{2}+t^{4}+\ldots=1 /\left(1-t^{2}\right)$. We see that, as in the $N=0$ case, the lacunary principle implies that $c_{2 \mathrm{R}}$ is perfect which in turn means that $P\left(Q_{2 R} ; t\right)=1 /\left(1-t^{2}\right)$. In fact, this is the same as the PP of $\mathrm{Q}_{0}$ (i.e. the $N=0$ space)! Thus, although $\mathrm{Q}_{0}$ and $\mathrm{Q}_{2 \mathrm{R}}$ can be topologically different spaces, they are homologically equivalent. So, one may recall the same $Q_{0}$ candidate spaces for $Q_{2 R}$ - loop space of SU(2), space of paths on $S^{3}$ joining two arbitrary points, $S^{2} \vee S^{4} \vee S^{6} \vee \ldots$, and $\mathrm{CP}^{\infty} \sharp 4$

\#3 We would like to thank C. Vafa for pointing out this crucial fact to us.

\#4 This latter space is, in fact, suggested by the relation between the minimal $N=2$ models and coset constructions of them based on $\mathrm{SU}(N) / \mathrm{SU}(N-1) \times \mathrm{U}(1) \approx \mathrm{CP}^{(N-1)}$. This was pointed out to us by $C$. Vafa.

\section{Acknowledgement}

The author would like to acknowledge many useful discussions with D. Birmingham, M. Blau, M. Greening, M. Mahdavi-H., A. Verjovsky, R. Vila Freyer and $\mathrm{R}$. Viswanathan. $\mathrm{He}$ is especially indebted to S. Randjbar-Daemi, C. Vafa and U. Yurtsever for invaluable contributions. Professor Abdus Salam, the International Atomic Energy Agency, UNESCO, and the International Center for Theoretical Physics, at Trieste, are acknowledged for their support.

\section{References}

[1] C. Vafa and N. Warner, Phys. Lett. B 218 (1989) 51; W. Lerche, C. Vafa and N. Warner, Nucl. Phys. B 324 (1989) 427;

E. Martinec, in: V.G. Knizhnik memorial volume, eds. L. Brink et al., to appear.

[2] T. Banks and E. Martinec, Nucl. Phys. B 293 (1987) 733.

[3] A.B. Zamolodchikov, JETP Lett. 43 (1986) 731; Sov. J. Nucl. Phys. 46 (1987) 1090.

[4] C. Vafa, Phys. Lett. B 212 (1988) 28.

[5] S. Das, G. Mandal and S.R. Wadia, Tata Institute preprint TIFR-TH-88/33 (May 1988).

[6] R. Bott, in: Recent developments in gauge theories, eds. G 't Hooft et al. (Plenum, New York, 1980); Bull. Amer. Math. Soc. (New Series) 3 (1980) 907; lectures Conf. in honour of René Thom (Paris, September 1988).

[7] A.B. Zamolodchikov, Sov. J. Nucl. Phys. 44 (1986) 529.

[8] D. Kastor, E. Martinec and S. Shenker, Nucl. Phys. B 316 (1989) 590

[9] K. Ito, ICTP preprint IC/89/49 (March 1989).

[10] V.I. Arnold, S.M. Gusein-Zade and A.N. Varchenko, Singularities of differentiable maps, Vol. 2 (Birkhäuser, Basel, 1985). 